\title{
Substance P (SP) Increases the Adhesion of Oral Squamous Cell Carcinoma (OSCC) To Human Umbilical Vein Endothelial Cells (HUVEC) Through Upregulating Adhesion Molecules; Implication for Metastasis
}

\section{Moustafa Elhousiny ${ }^{1 *}$, Kate Miller ${ }^{1}$, Anura Ariyawadana ${ }^{1,2}$, and Alan Nimmo $^{1}$}

${ }^{1}$ College of Medicine and Dentistry, James Cook University, Cairns, Australia

${ }^{2}$ Griffith Health Institute, Gold Coast Campus, Griffith University, Australia

*Corresponding Author: Moustafa Elhousiny, College of Medicine and Dentistry,

James Cook University, Cairns, Australia.

DOI: $10.31080 /$ ASDS.2020.05.1016
Received: December 02, 2020

Published: January 18, 2021

(C) All rights are reserved by Santhi G., et al.

\begin{abstract}
Objectives: Interaction of cancer cells with endothelial cells plays an important role in metastasis. Our study hypothesised that SP might play a role in the early onset adhesion of OSCC to HUVEC cells lines via upregulating the adhesion molecule expression.

Methods: CAL 27, SCC25, DOK, H157, BICR22 (OSCC) and HUVEC cell lines were used. Adhesion molecules' expression was measured by flowcytometry. The ECM645 adhesion assay was used for quantification of tumour-endothelial adhesion. Matrix metalloprotienease-2 (MMP-2) expression in OSCC cells was measured by total ELISA kit.

Results: Treatment with SP increased the adhesion of H157, CAL27 and DOK cells in a time-dependent manner, which was inhibited SP receptor antagonist. Monoclonal anti-adhesion antibodies inhibited the adhesion between OSCC and HUVEC. SP treatment increased (very late antigen-4) VLA-4 adhesion molecule in CAL27 and SCC25 cells and (lymphocyte function associated antigen-1) LFA-1 in BICR22. SP treatment increased MMP-2 release but it was not significant.

Conclusion: The study showed that SP could promote early onset oral cancer-endothelial cell adhesion. The study provided insights into the regulatory mechanism of this adhesion that it can occur in acute phase and in pre-cancer cells. These findings may provide potential new therapeutic targets for metastasis prevention.
\end{abstract}

Keywords: Oral Squamous Cell Carcinoma; Oral Cancer; Substance P; Tumour-endothelial Adhesion; Adhesion Molecules; NK-1R Antagonist

\section{Introduction}

OSCC, which represents $90 \%$ of all oral cavity tumours, is a locally invasive lesion with its average survival rate at 50\% [1]. Metastasis, whether local or distant, has shifted to becoming the prime killer of the cancer patient, not the original tumour itself [2]. The focus on exploring the mechanisms of cancer initiation has progressed the state of knowledge about primary tumours but added little to our understanding of how tumours can spread and kill the host [3]. The adhesion of tumour cells to endothelial cells represents a milestone event in the extravasation stage of metastasis $[4,5]$.
Insights into the malignant extravasation came from the recruitment of leukocytes in the inflammatory process [6]. The main similarity is that both processes are guided through localisation signals by the tumour, leukocytes or the endothelium, and responsible for promoting the adhesion molecule expression in both cells [7]. Several studies highlighted the key role of integrin (VLA-4, LFA) and selectin (SLeX) in the tumour- endothelial adhesion and metastasis of several cancers [8-11]. However, the upregulating signal and the exact role in eliciting an acute adhesion in oral cancer are poorly understood. 
SP was found to be significantly expressed in the tumour cell membrane, cytoplasm and nucleus of OSCC as well as the infiltrating lymphocytes of the neoplasms [12]. The expression of SP was found to be significantly associated with the presence of dysplasia and carcinoma in situ, which strongly suggested a role for SP in the early onset of oral oncogenesis [13]. Taking into consideration the main role of SP in inflammation (vessel permeability, adhesion and migration of leukocyte) and the up-regulated expression in OSCC, the study hypothesised that SP might play a role in the adhesion of OSCC cells to HUVEC cells. Therefore, the study aimed to examine the role of SP in stimulating OSCC-HUVEC adhesion as well as investigating the role of SP in upregulating the adhesion molecule expression of OSCC cell lines. The study also examined the effect of SP on tumour-endothelial adhesion and the effect of SP on the expression of MMP-2.

\section{Materials and Methods}

\section{Cell lines and cell culture}

Cells were selected to represent the progressive stages of oral cancer as follows: HOK primary oral keratinocyte isolated from human oral mucosa from Sciencell ${ }^{\circledR}$. CAL 27 (ATCC CRL- 2095) and SCC25 (ATCC CRL- 1628) tongue squamous carcinoma, both are from negative lymph node patients and were obtained from ATCC ${ }^{\circledR}$. A DOK cell line (ECACC- 94122104) represents mild to moderate dysplasia, H-157 ell line (ECACC-07030901) from oral squamouscell carcinoma of a positive lymph node patient and a BICR-22 cell line (ECACC- 04072106) from OSCC lymph node metastases were obtained from Sigma-Aldrich ${ }^{\circledR}$. Full description of culture media and cell culture of each cell line is provided in supplementary material (Appendix 1).

\section{Treatment}

Substance P acetate salt hydrate (SP) and [succinyl-ASP6, MEPHE8] Senktide Substance P analogue were purchased from SigmaAldrich $^{\mathrm{R}}$. Tumour necrosis factor (TNF- $\alpha$ ) was purchased from Sigma-Aldrich ${ }^{\circledR}$. A selective neurokinin receptor antagonist (NK-1R), CH123001 (N-(3,5-bis(trifluoromethyl)benzyl)-N-methyl-6-(4methylpiperazin-1-yl)-4(otolyl)nicotinamide) was a gift from $\mathrm{CH}$ Biotech Pty Ltd, Sydney, Australia.
Flow cytometry antibodies

Anti-integrin alpha 4 antibody CD49d PE (CAT No. 12-0499), Mouse IgG1, K isotype control (CAT No. 124714), Anti-CD11a antibody FITC (CAT No. BMS102FI) and Anti-CD15S Mouse antihuman Alexa Flour 647 (CAT No. 563526) were purchased from Affymetrix eBioscience ${ }^{\circledR}$.

\section{Adhesion molecule expression by flow cytometry}

Cells were adjusted to a concentration of $>5 \times 10^{4}$ cells $/ \mathrm{ml}$ as a single-cell suspension. Antibody cocktail was made at 1:20 concentration of each antibody with the FACS buffer with $50 \mu \mathrm{L}$ for each sample. $50 \mu \mathrm{L}$ of the antibody cocktail was added and the sample was incubated for at least 30 minutes at $4^{\circ} \mathrm{C}$ in the dark. The cells were washed three times with FACS buffer by centrifugation at 1500 RPM for 5 minutes and resuspended in $200 \mu \mathrm{l}$ to $1 \mathrm{ml}$ of icecold FACS buffer. The cells were kept in the dark on ice or at $4^{\circ} \mathrm{C}$ in a fridge until analysis by cell sorter (FACSCanto ${ }^{\mathrm{TM}}$ II, BD Biosciences) $(n=3)$.

\section{Cell adhesion assay}

HUVEC cells were harvested and seeded at a density of $5 \times 10^{4}$ in a 96-well floursence microplate (ECM645, Merckmillipore ${ }^{\circledR}$ ) or glass chamber slide and incubated for 48 hours at $37^{\circ} \mathrm{C}$. Similarly, tumour cells were seeded at $2 \times 10^{5}$ cells $/ \mathrm{ml}$. cells were starved for $12 \mathrm{~h}$ before adding the appropriate treatment to the corresponding tumour or endothelial cells (Appendix 2, experimental design). After treatment, both the endothelial cells and the tumour cell media were discarded and the tumour cells were treated with CalceinAM to a final concentration of $2.5 \mu \mathrm{M}$ for 30 minutes. Dye-loaded tumour cells from each well were co-incubated with the corresponding well of endothelial cells on the fluorescent plate for 30 minutes. Nonspecific binding was removed by washing each well 2-3 times with the assay buffer. The plate was read with a fluorescence plate reader using $485 / 530 \mathrm{~nm}$ excitation/emission filter sets $(\mathrm{n}=20)$. For the fluorescent microscope, images were obtained by Zeiss Axio Imager M1 and the imaging system (camera) is a CRI Nuance multispectral imaging system $(n=4)$. 
Cell adhesion was measured as a percentage of the basal adhesion according to the following:

Particular cell adhesion plate reader value $\quad$ X 100

Basal value plate value (non-treated cell adhesion value)

\section{ELISA assay for MMP-2 expression}

Media from different treated and untreated cells were collected and stored at $-20^{\circ} \mathrm{C}$ according to the manufacturer's manual. $50 \mu \mathrm{L}$ of assay diluents R1-116 (Quantikine Total MMP-2, R\&DSystems ${ }^{\circledR}$ ) was added to each well. $50 \mu \mathrm{L}$ of the standard, control or sample were added to each well and incubated on an orbital microplate shaker (0.12") orbit at 500RPM. After incubation, each well was washed three times with the wash buffer. $200 \mu \mathrm{L}$ of Total MMP2 conjugate was added to each well and incubated for another 2 hours. The washing procedure was repeated after incubation. 200 $\mu \mathrm{L}$ of substrate solution was added to each well and incubated for 30 minutes at room temperature in the dark. $50 \mu \mathrm{L}$ of stop solution was added. Immediately, the optical density of each well was determined using a microplate reader set to $450 \mathrm{~nm}$ and wavelength correction to 540-570 $\mathrm{nm}$. Samples were run in duplicates and repeated.

\section{Statistical analysis}

Student t-test was employed to determine the significance of the mean of two groups. ANOVA was used to determine the mean of more than two groups. The SPSS software package was used as the statistical analysis software. $\mathrm{P}<0.05$ was considered statistically significant.

\section{Results}

\section{Effect of Substance P on OSCC/HUVEC adhesion}

We examined the effect of SP on the adhesion of OSCC to HUVEC. All the treatments were done in two main groups. The first group had the treatment applied to the cancer cells, whereas the second group had HUVEC cells treated with the experimental reagents. The results showed that SP treatment increased the adhesion in H157, DOK and CAL27, whereas no effect was observed in either
SCC25 or BICR22 (Table 1). SP treatment $1 \mathrm{mcg} / \mathrm{ml}\left(10^{-6} \mathrm{M}\right)$ significantly increased the adhesion of OSCC cell lines in a time-course treatment from 1 to 4 hours (Figure 1). In both H157 and CAL 27 cells (Figure $1 \mathrm{~b}$ and $\mathrm{c}$ ), the adhesion levels were higher for the HUVEC treated group. Both groups had $(\mathrm{P}=0.001)$.

\begin{tabular}{|c|c|c|c|c|}
\hline \multirow{2}{*}{ Cell Line } & \multicolumn{4}{|c|}{ Time of Treatment/P Value for adhesion } \\
\cline { 2 - 5 } & 1-Hour & 2-Hour & 3-Hour & 4-Hour \\
\hline SCC25 & 0.84 & 0.21 & 0.59 & 0.95 \\
\hline BICR22 & 0.44 & 0.95 & 0.95 & 0.33 \\
\hline
\end{tabular}

Table 1: Shows the P- values for adhesion levels of SCC 25 and BICR22 OSCC cell lines to HUVEC endothelial cells at 1, 2, 3 and 4 hour-time when treated with SP $1 \mathrm{mcg} / \mathrm{ml}$.

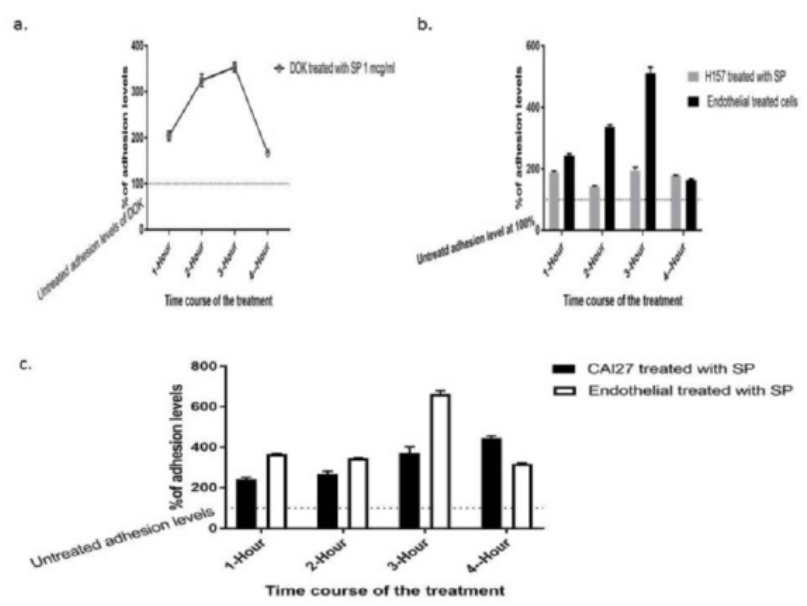

Figure 1: Effects of SP treatment on (a) DOK, (b) H157 and (c) CAL27 cells adhesion to HUVEC. Two experimental groups in which $\mathrm{H} 157$ cells were treated with $1 \mathrm{mcg} / \mathrm{ml}(10-6) \mathrm{M}$, where the other group had HUVECcells (b and c) treated with the same treatment. Treatment time was 1-4 hours and then both cells were incubated according to the assay protocol. Data are expressed as per cent of adhesion. Statistical analysis established the following significance: $\mathrm{P}<0.001$ vs control (untreated adhesion levels) for all the time points for either group, however, the group in which HUVEC cells were treated had higher values of adhesion levels $(n=20)$. 
Comparing SP and TNF- $\alpha$ effect on the adhesion of OSCC cell lines to HUVEC

Next, the effect of SP and TNF- $\alpha$ treatment (as a positive control) on the levels of adhesion in OSCC lines to HUVEC was compared. The results showed that both SP $1 \mathrm{mcg}\left(10^{-6}\right) \mathrm{M}$ and TNF- $\alpha 100 \mathrm{ng}$ $\left(10^{-9}\right)$ M stimulation for 3 hours significantly increased the adhesion levels of H157 and CAL 27 to HUVEC (Figure 2).

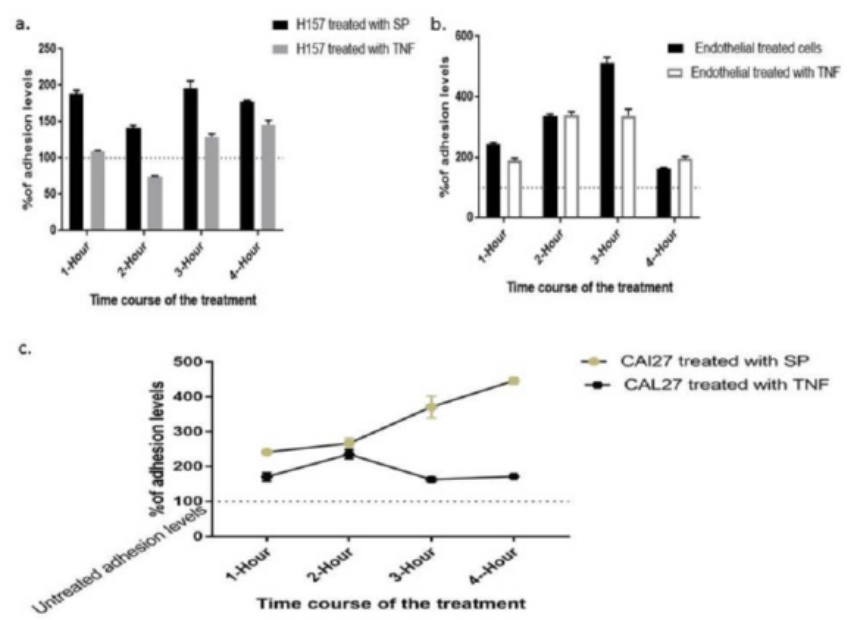

Figure 2: H157 tumour cell treated with either SP or TNF- $\alpha$ (a), H157 where the HUVEC cells treated (b) and CAL 27 (c) treated with SP $1 \mathrm{mcg}$ (10-6) M or TNF- $\alpha 100 \mathrm{ng}$ (10-9) M at 1-4 hours adhesion levels to HUVEC. Data are represented as per cent of adhesion levels vs control adhesion (untreated adhesion levels). $\mathrm{P}=$ 0.001 for all treatments. Error bars might be smaller than some data points.

\section{Effect of SP receptor (NK-1R) antagonist}

To investigate the effect of NK-1R antagonist on inhibiting the adhesion levels triggered by SP stimulation, we applied NK-1R antagonist to either the tumour cells or HUVEC. The data showed that the NK-1R antagonist significantly inhibited adhesion levels, reversing back to untreated baseline adhesion levels. Treating of H157 cells with SP treatment $1 \mathrm{mcg} / \mathrm{ml}$ for $1,2,3$ or 4 hours then treating the cells with $1 \mathrm{mcg} / \mathrm{ml}$ of the NK-1R antagonist for the same time point significantly inhibited $(\mathrm{P}=0.001)$ the adhe- sion levels with HUVEC cells at all the time points 1-4 hours. Additionally, treating the HUVEC cells with SP $1 \mathrm{mcg} / \mathrm{ml}$ at $1,2,3$ or 4 hours, then adding NK-1R, inhibited adhesion levels $(P=0.001)$ for all time points ((Figure 3a), which had had higher values of inhibition than H157 cells treated with NK-1R antagonist. In CAL27 cells, application of NK-1R antagonist to the cells significantly inhibited the adhesion levels compared with the same cells treated with SP only, with a maximum value of suppression at the 2-hour time point (Figure 3c). Also, the HUVEC cells treated with inhibitor suppressed adhesion significantly as compared to HUVEC cells treated with SP only, reaching a maximum at 3 hours ( $P=0.001)$

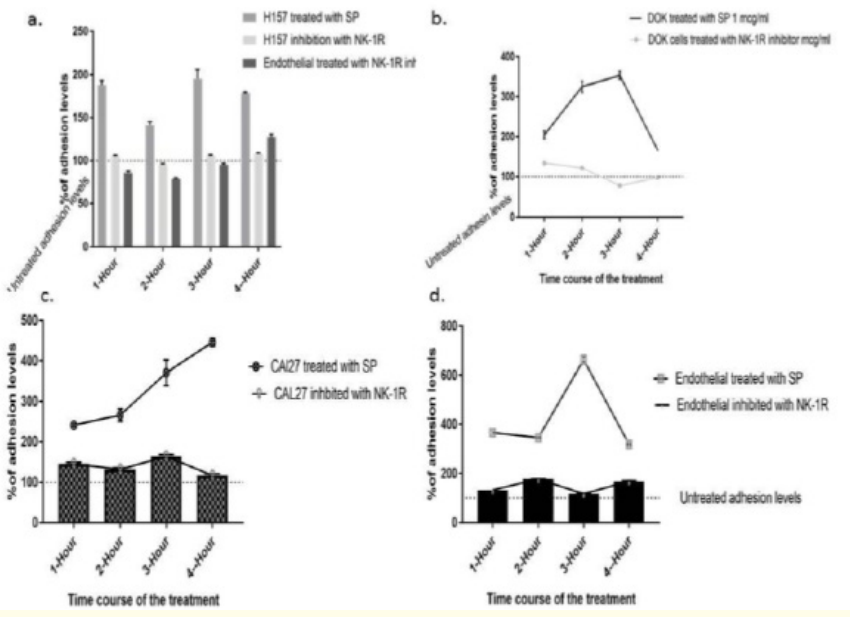

Figure 3: The effect of NK-1R antagonist on the inhibition of adhesion in H157, DOK and CAL27 to HUVEC cell. The effect of SP $1 \mathrm{mcg} / \mathrm{ml}$ treated $\mathrm{H} 157$ with the effect of the NK-1R $1 \mathrm{mcg} / \mathrm{ml}$ inhibitor applied to either H157 or HUVEC (Figure 3a). The effect of SP $1 \mathrm{mcg} / \mathrm{ml}$ treated DOK with the effect of the NK-1R $1 \mathrm{mcg} / \mathrm{ml}$ inhibitor applied to the same cells (Figure $3 \mathrm{~b}$ ). (Figure $3 \mathrm{c}$ and $3 \mathrm{~d}$ ) compare the effect of SP $1 \mathrm{mcg} / \mathrm{ml}$ treated CAL27 with the effect of the NK-1R $1 \mathrm{mcg} / \mathrm{ml}$ inhibitor applied to CAL27 and the also the effect of SP treatment and inhibitor on HUVEC cells on the adhesion to CAL 27. Data represented as per cent of adhesion levels vs control adhesion (untreated). $\mathrm{P}=0.001$ for all the time points (as a mean difference between SP treated and NK-1R inhibited). Some error bars are smaller than the corresponding data points and therefore they might not appear on the graph (All data points are represented as "Mean, SEM"). 
(Figure 3d). The suppression of those adhesion levels brought back the adhesion levels triggered by the SP treatment to values below the untreated adhesion threshold. Similarly, adhesion of DOK cells to HUVEC was significantly suppressed by the application of NK-1R to DOK cells with a maximum at 3 hours (Figure $3 \mathrm{~b}$ ).

Next, we performed a dose-response curve to investigate the effect of different concentrations of the NK-1R antagonist of the level of the adhesion. The NK-1R antagonist was used at concentrations of $100 \mathrm{ng}, 300 \mathrm{ng}, 750 \mathrm{ng}$ and $1 \mathrm{mcg}$ at time point 3-hour treatment (as this point was the average maximum for adhesion and inhibition for H157 and DOK). Application of increasing doses of NK-1R inhibitor to $\mathrm{H} 157$ cells produces a significant suppression of the adhesion which assumed a bell-shaped curve, with the maximum inhibitory point at $750 \mathrm{ng} / \mathrm{ml}$ and then the curve assumed a plateau (Figure 4a). A similar curve was observed in DOK cells (Figure $4 b)$.

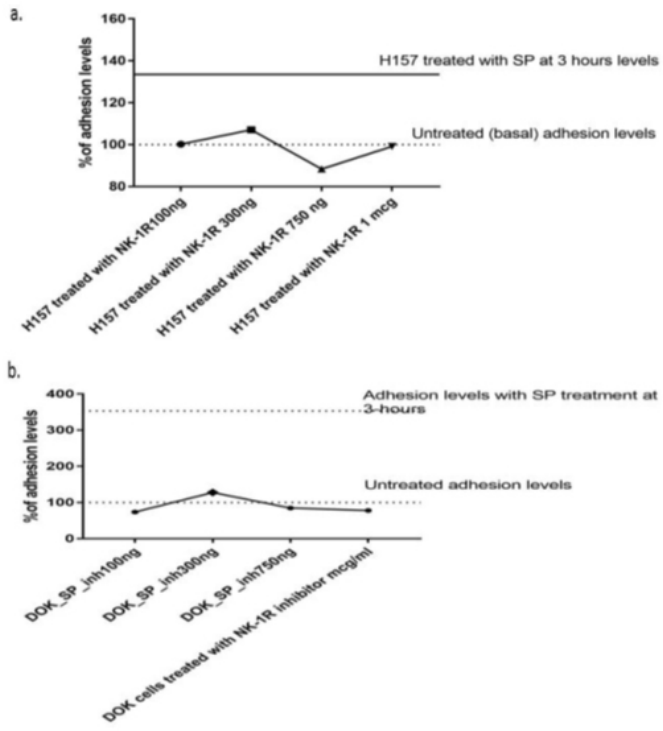

Figure 4: Dose-response curve of NK-1R antagonist at concentration $100 \mathrm{ng}, 300 \mathrm{ng}, 750 \mathrm{ng}$ and $1 \mathrm{mcg}$. (a) H157 cells adhesion levels at 3 hours showing SP stimulation levels above the untreated levels and the different concentration inhibition of adhesion by NK-1R, reversing the adhesion back to untreated basal levels. (b) DOK cells adhesion levels at 3 hours showing SP stimulation levels above the untreated levels and the different concentration inhibition of adhesion by NK-1R, reversing the adhesion back to untreated basal levels. Data are represented as per cent of adhesion levels versus control adhesion (untreated levels).
Effect of adhesion molecule anti-Human Monoclonal Antibodies on the adhesion

To investigate whether the monoclonal antibodies to the adhesion molecules had an effect on the adhesion levels stimulated by SP treatment, we used the adhesion monoclonal antibodies to Eselectin, vascular cell adhesion molecule (VCAM) and intercellular adhesion molecule (ICAM) (50 $\mathrm{g} / \mathrm{ml}$.) to test whether they can block the adhesion initiated with SP (Figure 5). Our data showed that in general, monoclonal antibodies to adhesion molecules suppressed the adhesion levels back to the untreated levels. However, the results were different for each cell line. In H157 cells, the maximum inhibitory effect was achieved with anti-E selectin followed by anti-ICAM which produced adhesion levels much lower than the threshold of the basal adhesion levels, while similar levels was observed for NK-1R inhibitor $(750 \mathrm{ng} / \mathrm{ml})$ as well as anti-VCAM antibody at levels close to the basal untreated adhesion levels. In DOK precancer cells, the situation was completely reversed with the most potent adhesion suppressors NK-1R antagonist and antiVCAM Mab.

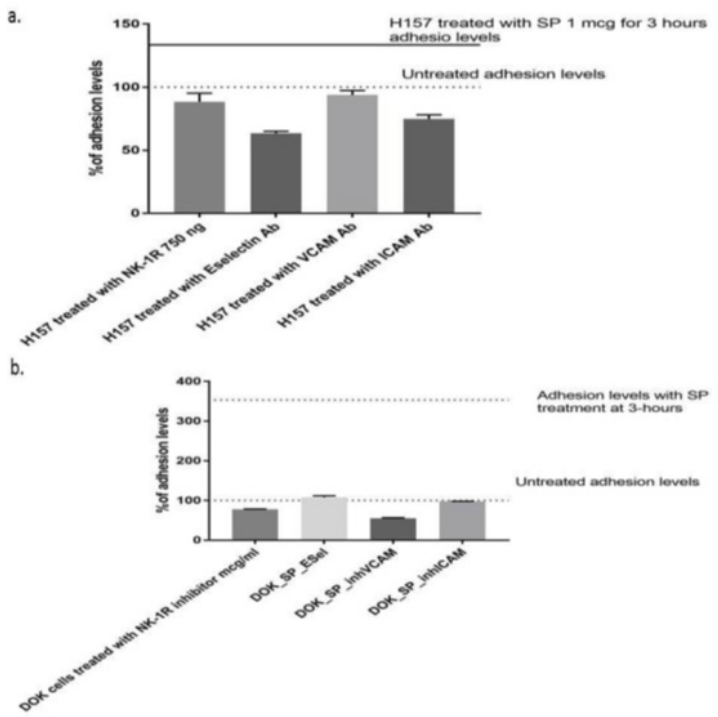

Figure 5: Comparing the effect of adhesion molecule monoclonal antibodies on the adhesion levels triggered by SP treatment to the NK-1R antagonist at time point 3 hours. (a): H157 cell line. (b): DOK cell lines. 


\section{Effect of SP treatment on the adhesion molecule expression}

We hypothesised that surface adhesion molecules should be the main player in the adhesion event and that the increase in cell adhesion is attributable to induction of adhesion molecule expression on stimulated cells by either SP treatment. We used flow cytometry to analyse the expression of adhesion ligands (CD49, CD15s, CD11a) for HUVEC adhesion receptors VCAM, E-selectin and ICAM, respectively in each cell line.

\section{Basal levels of adhesion molecule expression in OSCC}

First, we investigated the initial levels of those expression molecules in each cell line, according to the malignant potential, form normal, precancer, primary tumour (with/without lymph node metastases) to metastases. The untreated levels of adhesion molecule expression were plotted according to the mean value of the experimental samples for CD49, CD15s and CD11, respectively (Figure 6). Our results showed that each adhesion molecule expression increased significantly in direct proportion to the increase in the malignant potential of the cell line, ranging from a negligible expression in the normal oral keratinocyte to the more aggressive H157 cell line with positive lymph node, and then declined in the metastases cell line BICR22.

\section{Effect of SP treatment on the expression of adhesion molecules}

Our hypothesis was that if SP increased adhesion levels and, given the trend of the expression of adhesion molecules in the different cell lines, SP treatment may trigger expression of these adhesion molecules. Therefore, we performed a time-course treatment of SP 1 mcg at 1-36 hours and analysed the expression of each adhesion molecule in each cell line using the FACS cell sorter. Our results showed that SP treatment increased the expression of CD49 in CAL27 in a time-course treatment (Figure 7a and 7b).

The peak of expression increase was at 3 hours treatment and then the expression reverted to untreated levels. The 3-hour treatment significantly increased the expression of both CD11 FITC and CD49 molecules as indicated by FACS analysis in both SCC25 and BICR22, respectively (Figure 7b and 7c).

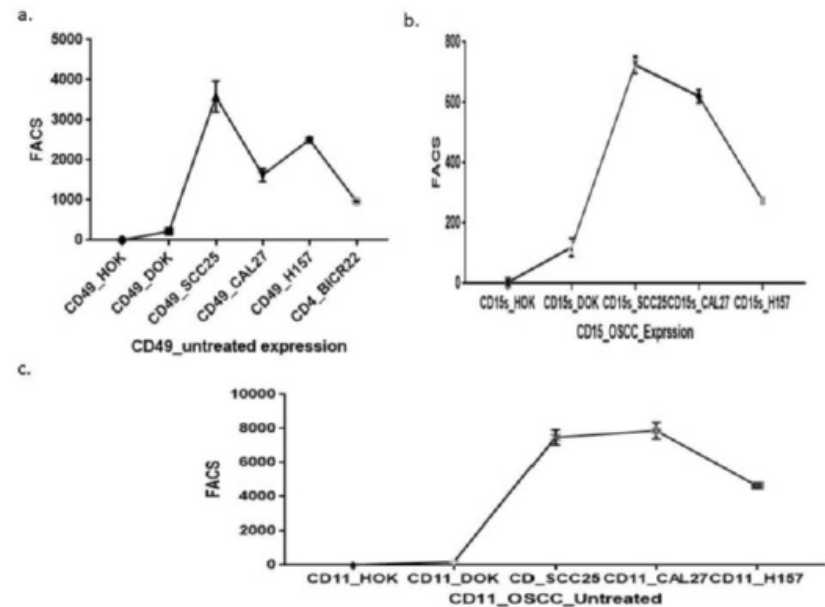

Figure 6: (a) Untreated CD49 adhesion molecule expression levels. (b) Untreated CD15s adhesion molecule expression levels (c) Untreated CD11 adhesion molecule expression levels in different stages of OSCC cell lines as illustrated by mean fluorescence intensity using FACS analysis.

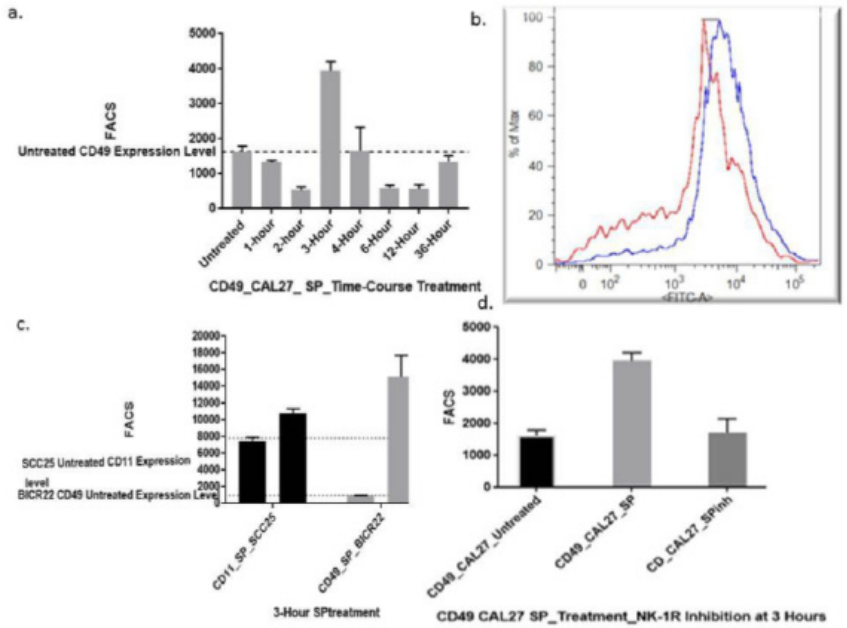

Figure 7: (a) Mean values of VlA-4 (CD49) adhesion molecule expression ( $\mathrm{n}=3)$ for CAL 27 cell lines treated with SP 1-36 hours. (b) FACS histogram showing CD11 FITC in SCC25 before and after treatment. (c) Effect of SP treatment at 3 hours on the expression of adhesion molecules CD11 and CD49 in both SCC25 and BICR22 OSCC cell lines, indicating the untreated levels. (d) The expression of CD49 in CAL27 cells (untreated levels, treated for three hours, and inhibited with NK-1R antagonist) as shown by the FACS analysis. 
The effect of the NK-1R antagonist on the expression of adhesion molecules stimulated by SP treatment.

Finally, we investigated the effect of the NK-1R antagonist on the expression of adhesion molecules in OSCC cell lines, as we predicted that it would negate the stimulation caused by SP treatment $1 \mathrm{mcg}$. The inhibitor produced an inhibitory effect on CD49 (VLA-4) integrin in CAL27 at 3 hours adhesion in which it reverted the expression levels back to untreated levels (Figure 7d). Interestingly, the NK-1R antagonist inhibited the effect of TNF- $\alpha$ stimulation to CD49 in the BICR22 cell line $(\mathrm{P}=0.002)$.

\section{Fluorescence microscope}

Using the same protocol as the endothelial adhesion assay, we investigated the effect of SP and its inhibitor NK-1R antagonist on the adhesion of DOK and H157 OSCC cell lines to HUVECs on a chamber glass with the fluorescent microscope. The result showed that the adhesion of both cell lines to HUVEC increased significantly with SP stimulation at 3 hours (Figure 8). By contrast, when cells were treated with NK-1R inhibitor for 3 hours, it showed significant reduction of this adhesion to HUVEC $(n=20)$.

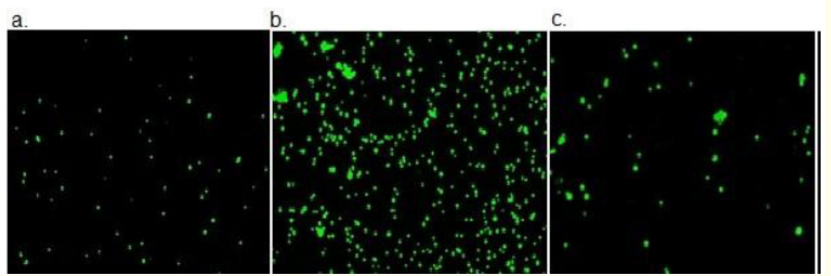

Figure 8: (a) Untreated basal adhesion levels of $\mathrm{H} 157$ to HUVEC cell lines. (b) SP treatment at 3 hours adhesion levels of H157 to HUVEC. (c) Inhibition of adhesion of H157 to HUVEC by NK-1R antagonist.

\section{Effect of SP and TNF- $\alpha$ on the release of MMP-2}

Finally, we investigated the effect of SP and TNF- $\alpha$ treatment on the release of MMP-2 in OSCC cell lines. The experimental design involved measuring MMP-2 levels at basal (untreated levels) as well as after treatment at the 3-hour time point using the ELISA technique. Neither SP nor TNF- $\alpha$ treatment significantly affected the expression (results shown in table 2). However, some interesting findings have been obtained. Firstly, the untreated expression of OSCC cell lines assumed an increasing expression of MMP-2 with the exception of DOK premalignant cells, which remarkably showed higher baseline expression of MMP-2 at a mean $1.76 \mathrm{ng} /$ $\mathrm{ml}$. This expression was proportional to the malignant potential of the corresponding cell lines. Secondly, SP treatment significantly decreased the expression of MMP-2 in BICR22 metastatic cell line $(\mathrm{P}=0.003)$.

\begin{tabular}{|c|c|c|}
\hline Cell Line & Basal Expression & Treatment/P Value \\
\hline DOK & $++1.76 \mathrm{ng} / \mathrm{ml}$ & SP: 0.36 TNF: 0.82 \\
\hline CAL27 & $+1.91 \mathrm{ng} / \mathrm{ml}$ & SP: 0.45 TNF: 0.12 \\
\hline SCC25 & $++1.51 \mathrm{ng} / \mathrm{ml}$ & SP: 0.92 TNF: 0.98 \\
\hline H157 & $++.23 . \mathrm{ng} / \mathrm{ml}$ & SP: 0.32 TNF: 0.23 \\
\hline BICR22 & $+++1.85 \mathrm{ng} / \mathrm{ml}$ & SP: $0.04^{*}$ TNF: 0.78 \\
\hline
\end{tabular}

Table 2: MMP-2 expression in OSCC cell lines before and after treatment of either SP or TNF- $\alpha$ using ELISA assay.

\section{Discussion}

Metastasis, the main cause of cancer patient deaths, starts with the tumour cell losing adhesion with its neighbouring cells, invading the surrounding tissues, gaining access to blood vessels, and finally adhering to and exiting the blood vessels to implant in secondary sites [14]. Interaction of cancer cells with endothelial cells plays an important role in both tumour angiogenesis and metastasis [15]. Recent studies support an important role for inflammation in cancer initiation, progression and metastasis. In addition, emerging studies highlight inflammation as a main driver of the different stages of metastasis, such as tumour-endothelial cell adhesion [16]. However, the current literature has not identified the particular mechanism of the relationship of inflammation of metastasis or the precise effect of inflammatory mediators on adhesion and extravasation of tumour cells.

Our results showed that: 1 ) SP and TNF- $\alpha$ increased the adhesion of H157, CAL27 and DOK cells to HUVEC endothelial cells in a time-course treatment with a peak adhesion at 3 hours; 2) the 
endothelial treated group had higher adhesion values than the tumour treated groups; 3) NK-1R inhibitor reduced adhesion in a time and dose-dependent manner; and 4) Monoclonal anti-adhesion antibodies inhibited adhesion between OSCC and HUVEC.

These observations demonstrate a new observation of cancerendothelial cell adhesion, occurring in the acute phase, which may be of fundamental importance to the process of metastasis. Firstly, early tumour-endothelial cell adhesion may contribute to enhanced tumour cell survival via bypassing immune attack and loss of attachment $[17,18]$. Secondly, it provides tumour cells with an early seeding capacity. In fact, early adhesion has been found to increase the metastatic capability of cancer cells [19]. Cancer-endothelial cell adhesion has been speculated to be related to cancer progression and metastasis via a number of studies. However, the mechanisms of adhesion between cancer cells and endothelial cells and its main drivers remain unclear.

There has been a lack of research into the triggers and mechanisms underlying cancer-endothelial adhesion in an acute phase. Inflammation is a cardinal characteristic of the tumour microenvironment and plays multifaceted and critical roles in every aspect of tumour development and progression [20]. In the present study, we demonstrated for the first time that the inflammatory mediators, SP $1 \mathrm{mcg} / \mathrm{ml}$ and TNF- $\alpha-\alpha 100 \mathrm{ng} / \mathrm{ml}$ were involved in oral cancer-endothelial cell adhesion in acute phase manner with a peak of 3 hours in-vitro.

It is well established that SP acts as a mitogenic stimulator for tumour growth and proliferation of tumour cells [21-23]. Brener., et al. described SP expression in infiltrating lymphocytes in $81.6 \%$ ( $n=71)$ and in peritumoural or intratumoural blood vessels in $58.1 \%(n=43)$, where they proposed a diffusion mechanism for SP from lymphocytes to the parenchyma of the tumour [17]. Similarly, Munoz., et al. identified NK-1R receptors in smooth muscle of the blood vessels surrounding the primary malignant melanoma [24]. Similar results were obtained in leukaemia cell lines [25]. In all those studies, the NK-1R antagonist inhibited the growth of tumour cells, suggesting a potent mitogenic action for SP/NK1R complex interaction, where several mechanisms have been proposed. The SP/NK-1R complex activates different mitogen activated protein kinase (MAPK) signalling cascades such as extracellularsignal-regulated kinase (ERK) and P38 MAPK, through forming a scaffolding complex with $\beta$-arrestin [26]. Additionally, the SP/NK$1 \mathrm{R}$ complex can mediate other pathways such as the nuclear factor kappa-light-chain-enhancer of activated B cells (NF-k $\beta$ ) pathways [27], and transactivates other tyrosine kinase receptors such as epidermal growth factor receptor (EGFR). In one recent study, Carotid artery injection of Walker 256 cells (for experimental metastasis induction) increased SP reactivity in coincidence with the primary tumour invasion of brain microvessels [28]. These results can be explained in the context of SP acts as a mitogenic inducer of a mutant phenotype that selects for more invasive subpopulations of tumour cells with enhanced growth and migratory abilities that follow a concentration gradient, providing a pathway for these cells' invasion.

Based on previous observation that the close contact and adhesion of cells is a prerequisite for cell transmigration [18], we hypothesised that adhesion molecules are the main requirement of the adhesion process. Firstly, the adhesion molecule expression followed a predicted pattern which increased from no expression in the normal oral keratinocytes to the lymph node positive cell line H157 and declined in the metastatic cell lines (for the three adhesion molecules). The precancer cell line DOK had an elevated expression profile which agrees with previous studies, suggesting an early invasive/metastatic phenotype in the tumour cycle [29].

Although SP treatment increased a specific adhesion molecule in each cell line as observed by FACS, the VLA- 4 ( $\alpha 4 \beta 1$ integrin) pathway was the main molecule affected with the SP treatment and may mediate the enhanced oral cancer-endothelial cell adhesion in this study. Rebhun., et al. reported that B16 $\alpha 4+$ tumours generated lymph node metastases in $80 \%$ of mice and that melanoma cells that were deficient in $\alpha 4$ integrins (B16 $\alpha 4-$ ) were non-tumorigenic [30]. Okahara., et al. showed that interaction between VLA-4 on tumour cells and VCAM-1 on endothelial cells is implicated in progression of metastasis in-vivo [16]. Collectively, these data show that the $\alpha 4$ integrin expressed by cancer cells contributes to tumorigenesis and may also facilitate metastasis to regional lymph nodes by promoting stable adhesion of tumour cells to the lymphatic vasculature. It has been reported that cancer cell-endothelial adhe- 
sion is thought to be regulated by the mechanical properties of the cancer cells and also by the specific expression of various adhesion molecules and/or ligands to adhesion molecules on the surface of cancer cells and endothelial cells induced by inflammatory mediators [31-33]. Thus, these results provided preliminary evidence for the adhesion of oral cancer cells to endothelial cells, suggesting a role for VLA-4 in this adhesion.

Our study reports a number of interesting findings; firstly that DOK precancer cells treatment with SP has produced adhesion results comparable with H157 positive lymph node cells. This indicates an early invasive phenotype in the tumour life cycle predisposing to an early metastatic potential. Secondly, although NK-1R inhibitor suppressed the adhesion, it was not as effective in suppressing the adhesion molecule expression, except in CAL 27 and BICR22. In addition, the monoclonal human antibodies to adhesion molecules had a profound inhibitory effect on the adhesion. Those results will require further investigation to underpin the exact mechanism of the tumour-cell interaction. Thirdly, the endothelial treated group with either SP or NK-1R inhibitor had more significant values of adhesion stimulation or adhesion suppression than the tumour-treated group in most cases. We suggest that this may be explained in the context of organ-specific metastasis. Finally, the NK-1R antagonist inhibited not only SP treatment but also TNF- $\alpha$ treatment which might be due to transactivation or for the suppression of downstream pathways, as SP and TNF- $\alpha$ are known to activate downstream NF-K $\beta$ pathway [27].

Adhesion of tumour cells to endothelial cells requires subsequent steps to promote tumour cell migration and implantation in the secondary site. The MMP group of enzymes has been implicated in facilitating the tumour cell migration and invasion of the endothelial barrier [34]. In our study, OSCC cell lines had a varying basal level of expression of MMP-2, which was directly proportional to its malignant potential. However, the treatment of SP or TNF- $\alpha$ did not significantly affect this expression. More importantly, the treatment of the BICR 22 cell line with SP treatment for 3 hours significantly decreased this expression significantly $(P=0.04)$. Additionally, the DOK premalignant cell line had a high expression of MMP-2 which again suggests an early metastatic potential in tumour cycle [29]. Li., et al. reported a significant effect of SP on the expression of MMP-2 on mRNA level [35]. This data will require further investigation to validate those results.

\section{Limitations}

The current study was performed in-vitro, using OSCC cell lines and this approach, despite providing an easy experimental approach, neglects the tumour microenvironment. Also, there is a limitation in the small sample size but the study provides a preliminary dataset that can be used for further validation in either animal or clinical studies.

\section{Conclusion}

The present study inflammatory mediator SP could promote oral cancer-endothelial cell adhesion. The SP enhanced oral cancerendothelial cell adhesion can occur in an early onset and might be mediated through the VCAM-1/VLA-4 pathway. This adhesion was significantly suppressed with monoclonal antibodies to adhesion molecule as well as the NK-1R antagonist. These findings may provide insights into tumour microenvironment interaction and reveal new therapeutic targets for cancer prevention and treatment.

\section{Conflict of Interest}

No potential conflict of interest declared.

\section{Acknowledgment}

This work was a part of the corresponding author $\mathrm{PhD}$ thesis and was supported by the College of Medicine and Dentistry, James Cook University, QLD, Australia (Fund. 2132/1101/0104SE0.920102- FoR.111201 ) ethical approval permit No. JCUCD2014-008.

Special thanks for QTHA lab scientists: they were of great help in the experimental work from cell culture all the way up. I would like to thank Dr. John Cokley for his efforts in language editing and proofing this work.

\section{Author Contribution}

All authors contributed to the study conception and design. Material preparation, data collection and analysis were performed 
by [Moustafa Elhousiny], [kate Miller] and [Alan Nimmo]. Project administration, funding and supervision was performed by [Alan Nimmo], [Anura Ariyawadana] and [Kate Miller]. Resources were provided by [Alan Nimmo]. The first draft of the manuscript was written by [Moustafa Elhousiny] and all authors commented on previous versions of the manuscript. All authors read and approved the final manuscript for publication.

\section{Bibliography}

1. Warnakulasuriya S. "Global epidemiology of oral and oropharyngeal cancer". Oral Oncology 45.4 (2009): 309-316.

2. Ord R and BLANCHAERT R. "Current management of oral cancer: A multidisciplinary approach". The Journal of the American Dental Association 132 (2001): 19-23.

3. Chiang A and Massagu'e J. "Molecular basis of metastasis". New England Journal of Medicine 359.26 (2008): 2814-2823.

4. Lam L., et al. "Retrospective study of survival and treatment pattern in a cohort of patients with oral and oropharyngeal tongue cancers from 1987 to 2004". Oral Oncology 43.2 (2007): 150-158.

5. Sargeran K. “Oral Cancer in Tehran, Iran: An approach for understanding disease burden". Helsingin yliopisto (2008).

6. Wu Y and Zhou B. "Inflammation: a driving force speeds cancer metastasis". Cell Cycle (Georgetown, Tex) 8.20 (2009): 3267.

7. Entschladen F., et al. "Tumor-cell migration, invasion, and metastasis: navigation by neurotransmitters". The Lancet Oncology 5.4 (2004): 254-258.

8. Sawada R., et al. "Differential E-selectin-dependent adhesion efficiency in sublines of a human colon cancer exhibiting distinct metastatic potentials". Journal of Biological Chemistry 269.2 (1994): 1425-1431.

9. Fukami A., et al. "Macrosphelide B suppressed metastasis through inhibition of adhesion of sLe $(\mathrm{x}) /$ E-selectin molecules". Biochemical and Biophysical Research Communications 291.4 (2002): 1065-1070.

10. Wang S., et al. "Effect of an anti-CD54 (ICAM-1) monoclonal antibody (UV3) on the growth of human uveal melanoma cells transplanted heterotopically and orthotopically in SCID mice". International Journal of Cancer 118.4 (2006): 932-941.
11. Schlesinger M and Bendas G. "Vascular cell adhesion molecule-1 (VCAM-1)-An increasing insight into its role in tumorigenicity and metastasis". International Journal of Cancer (2014).

12. Brener S., et al. "A role for the substance P/NK-1 receptor complex in cell proliferation in oral squamous cell carcinoma". Anticancer Research 29.6 (2009): 2323-2329.

13. Gonzalez-Moles MA., et al. "Substance P and NK-1R expression in oral precancerous epithelium". Oncology Reports 22.6 (2009): 1325-1331.

14. Martin TA., et al. "Cancer Invasion and Metastasis: Molecular and Cellular Perspective". In: Madame Curie Bioscience Database Internet. Austin (TX): Landes Bioscience; 2000-2013.

15. Orr FW., et al. "Interactions between cancer cells and the endothelium in metastasis". The Journal of Pathology 190.3 (2000): 310-329.

16. Okahara H., et al. "Involvement of very late activation antigen 4 (VLA-4) and vascular cell adhesion molecule 1 (VCAM-1) in tumor necrosis factor $\alpha$ enhancement of experimental metastasis". Cancer Research 54.12 (1994): 3233-3236.

17. Fuster M and Esko J. "The sweet and sour of cancer: glycans as novel therapeutic targets". Nature Reviews Cancer 5.7 (2005): 526-542.

18. Kobayashi H., et al. "Endothelial cell adhesion molecules and cancer progression". Current Medicinal Chemistry 14.4 (2007): 377-386.

19. Strilic B and Offermanns S. "Intravascular Survival and Extravasation of Tumor Cells". Cancer Cell 32.3 (2017): 282-293.

20. Szebeni GJ., et al. "Inflammation and Cancer: Extra- and Intracellular Determinants of Tumor-Associated Macrophages as Tumor Promoters". Mediators of Inflammation (2017): 13.

21. Shah I., et al. "Clinical stage of oral cancer patients at the time of initial diagnosis". Journal of Ayub Medical College, Abbottabad 22.3 (2010): 61-63.

22. Pulte D and Brenner H. "Changes in survival in head and neck cancers in the late 20th and early 21st century: a period analysis". The Oncologist 15.9 (2010): 994-1001. 
23. Van der Waal I. "Are we able to reduce the mortality and morbidity of oral cancer; some considerations". Medicina Oral, Patologia Oral Y Cirugia Buccal 18.1 (2013): 33.

24. Muñoz M., et al. "The NK-1 receptor is expressed in human melanoma and is involved in the antitumor action of the NK-1 receptor antagonist aprepitant on melanoma cell lines". Laboratory Investigation 90.8 (2010): 1259.

25. Nowicki M., et al. "The predicting role of Substance P in the neoplastic transformation of the hypoplastic bone marrow". Journal of Clinical Pathology 59.9 (2006): 935-941.

26. DeFea KA., et al. "The proliferative and antiapoptotic effects of substance $P$ are facilitated by formation of a beta -arrestindependent scaffolding complex". Proceedings of the National Academy of Sciences of the United States of America 97.20 (2000): 11086-11091.

27. Sun J., et al. "Substance P enhances NF-kappa $\beta$ transactivation and chemokine response in murine macrophages via ERK1/2 and p38 MAPK signaling pathways". American Journal of Physiology Cell Physiology 294.6 (2008): C1586-1596.

28. Lewis K. "The role of substance $\mathrm{P}$ in the progression and complications of secondary brain tumors 2012.

29. Friberg S, Nystrom A. Cancer Metastases: Early Dissemination and Late Recurrences". Cancer Growth and Metastasis 8 (2015): CGM.S31244.

30. Rebhun RB., et al. "Constitutive expression of the alpha4 integrin correlates with tumorigenicity and lymph node metastasis of the B16 murine melanoma". Neoplasia 12.2 (2012): 173182.

31. Liang S and Dong C. "Integrin VLA-4 enhances sialyl-Lewis-x/a-negative melanoma adhesion to and extravasation through the endothelium under low flow conditions". American Journal of Physiology-Cell Physiology 295.3 (2008): C701C707.

32. Klemke M., et al. "High affinity interaction of integrin $\alpha 4 \beta 1$ (VLA-4) and vascular cell adhesion molecule 1 (VCAM-1) enhances migration of human melanoma cells across activated endothelial cell layers". Journal of Cellular Physiology 212.2 (2017): 368-374.
33. Li H., et al. "HIF-1alpha-activated ANGPTL4 contributes to tumor metastasis via VCAM-1/integrin beta1 signaling in human hepatocellular carcinoma". Hepatology 54.3 (2011): 910-919.

34. Deryugina EI and Quigley JP. "Matrix metalloproteinases and tumor metastasis". Cancer Metastasis Review 25.1 (2006): 9-34.

35. Li X., et al. "Neurotransmitter substance P mediates pancreatic cancer perineural invasion via NK-1R in cancer cells". Molecular Cancer Research: MCR 11.3 (2013): 294-302.

\section{Assets from publication with us}

- Prompt Acknowledgement after receiving the article

- Thorough Double blinded peer review

- Rapid Publication

- Issue of Publication Certificate

- High visibility of your Published work

Website: www.actascientific.com/

Submit Article: www.actascientific.com/submission.php

Email us: editor@actascientific.com

Contact us: +919182824667 